\title{
Correction to: Mercury accumulation, speciation, and temporal trends in Atlantic Stingrays (Hypanus sabinus)
}

\author{
Brianne K. Soulen ${ }^{1} \cdot$ Douglas H. Adams ${ }^{2}$ - Aaron P. Roberts ${ }^{1}$
}

Published online: 15 April 2019

(c) Springer Science+Business Media, LLC, part of Springer Nature 2019

Correction to: Ecotoxicology (2019) 28:251-260 https://doi.org/10.1007/s10646-018-02014-w

The original version of the article was published with a wording error.

The corrections are: In the 2nd sentence of the Statistical Analyses section, the current sentence lacks important statistical assumption wording.
The sentence in the Online First PDF reads: "All data were tested for normality and appropriate test were used where assumptions were not met."

It should read (as in the earlier original version):

"All data were tested for homoscedasticity and normality, and data were transformed when assumptions were not met."
The original article can be found online at https://doi.org/10.1007/ s10646-018-02014-w.

Brianne K. Soulen

brianne.soulen@unt.edu

1 Department of Biological Sciences and Advanced Environmental Research Institute, University of North Texas, Denton, TX, USA

2 Florida Fish and Wildlife Conservation Commission, Fish and Wildlife Research Institute, Indian River Field Lab,

Melbourne, FL, USA 